\title{
Segurança do paciente: notificação de incidentes na Unidade de Terapia Intensiva
}

\author{
Patient safety: notification of incidents in the \\ Intensive Care Unit
}

\section{Marcela Vieira Lordelo', Glicia Gleide Gonçalves Gama}

'Autora para correspondência. Escola Bahiana de Medicina e Saúde Pública. Salvador, Bahia, Brasil. ORCID: 0000-0003-1714-2964. msv.enf@gmail.com ${ }^{2}$ Escola Bahiana de Medicina e Saúde Pública. Salvador, Bahia, Brasil. ORCID: 0000-0002-0221-0453.gliciaggama@bahiana.edu.br

RESUMO I OBJETIVO: descrever a notificação de incidentes na unidade de terapia intensiva após implementação do Programa Nacional de Segurança do Paciente. MÉTODO: estudo retrospectivo, do tipo descritivo de abordagem quantitativa, realizado na unidade de terapia intensiva de um Hospital Privado do município de Salvador, Bahia, Brasil, nos meses de novembro e dezembro de 2016. RESULTADOS: a amostra de incidentes foi de 210 notificações, que revelaram $80 \%(n=168)$ por erro de medicação. Foram encontradas $11 \%(n=23)$ notificações por exteriorização de dispositivos; $4,8 \%(n=10)$ por flebite e um total de $4,3 \%(n=9)$ referentes à lesão por pressão. Não existiram notificações de queda no período estudado. CONCLUSÃO: O levantamento das notificações dos incidentes, mesmo existindo subnotificações, evidencia - tipo de atendimento prestado pela organização e a preocupação com a segurança do paciente.

DESCRITORES: Segurança do paciente. Erros médicos. Unidades de Terapia Intensiva. Erros de medicação. Flebite.

\begin{abstract}
OBJECTIVE: To describe incident reporting in the intensive care unit after implementation of the National Patient Safety Program. METHOD: retrospective, descriptive study of a quantitative approach performed at the intensive care unit of a Private Hospital in the city of Salvador, Bahia, Brazil, in the months of November and December 2016. RESULTS: the incident sample was 210 notifications, which revealed $80 \%(n=168)$ due to medication error. We found $11 \%(n=23)$ notifications for device exteriorization; $4.8 \%$ $(n=10)$ for phlebitis and a total of $4.3 \%(n=9)$ for pressure injury. There were no reports of falls in the study period. CONCLUSION: The survey of incident reports, even if there are underreporting, shows the type of care provided by the organization and the concern with patient safety.
\end{abstract}

DESCRIPTORS: Patient safety. Medical errors. Intensive Care Units. Medication errors. Phlebitis. 


\section{Introdução}

O termo segurança do paciente é definido como redução dos riscos de danos desnecessários associados à assistência em saúde até um mínimo aceitável, reconhecidos como eventos adversos provocados pela assistência ao paciente sem relação à evolução da doença de base, gerando lesões, óbito ou aumento dos dias de internação hospitalar. $O$ incidente segundo a Classificação Internacional de Segurança do Paciente (International Classification for Patient Safet - ICPS), é um evento ou circunstância que poderia ter resultado ou resultou em dano desnecessário ao paciente, advindos de atos não intencionais ou intencionais'.

Sendo assim, o risco está relacionado à chance de acontecer um evento que poderá ser danoso para saúde, direta ou indiretamente, podendo ser associado a produto, ambiente, serviço e processo'.

A mortalidade atribuída aos eventos adversos na assistência à saúde está classificada como $8^{a}$ causa de mortalidade nos EUA ${ }^{2}$. O Brasil teve 31.774 incidentes notificados no ano de $2015^{3}$. A Organização Mundial da Saúde (OMS), em 2005, lançou a Aliança Mundial para Segurança do Paciente. O objetivo era chamar atenção para o tema como um problema global de saúde, despertando a consciência e enfoque político para alcançar melhorias na segurança à assistência e apoiar os países no desenvolvimento de políticas públicas e práticas para segurança do paciente em todo o mundo ${ }^{1,3}$.

Pela Lei Orgânica da Saúde (Lei n8.080, 19 de setembro de 1990) o Sistema Único de Saúde é responsável por definir e coordenar o sistema de vigilância sanitária, controlar e fiscalizar produtos, substância de interesse para a saúde e procedimentos, estando diretamente relacionado ao Sistema Nacional de Vigilância Sanitária e a saúde da população ${ }^{4}$. No Brasil, o Projeto de Avaliação de Desempenho de Sistemas de Saúde (Proadess) propõe uma metodologia de avaliação de desempenho para o País, considerando a segurança como parte do cuidado em saúde com qualidade, na identificação, minimização dos possíveis riscos, apresentando definições e indicadores para cada dimensão ${ }^{5}$.
O Ministério da Saúde institui o Programa Nacional de Segurança do Paciente, pela Portaria $n^{\circ} 529$, de $1^{\circ}$ de abril de $2013^{4}$. No mesmo ano lança a RDC $n^{\circ} 36$, de 25 de julho de 2013, com objetivo de instituir ações para a promoção da segurança do paciente em serviços de saúde e a melhoria da qualidade nos serviços de saúde 6 .

O enfermeiro tem um papel importante dentro do contexto da segurança do paciente por estar envolvido em todas as etapas do cuidado e na função gerencial da unidade, este profissional oferece a qualidade da assistência de enfermagem garantindo um atendimento integral, com aprimoramento cada vez maior a partir dos indicadores de qualidade do serviço, reduzindo as chances de acontecer eventos adversos ${ }^{7}$. A atuação dos profissionais de saúde é multiprofissional, os quais devem exercer funções de esclarecer dúvidas, garantir uma assistência completa ao paciente e familiar a partir do conhecimento técnico e científico, buscando contribuir na sua recuperação. Sendo o enfermeiro, responsável por ouvir as necessidades da família, gerenciar e coordenar o processo assistencial desenvolvido no contexto da instituição hospitalar ${ }^{8}$.

A Unidade de Terapia Intensiva (UTI) é um setor complexo do hospital que possui pacientes instáveis hemodinamicamente e que demandam ação imediata, pelo risco iminente de morte. A utilização de equipamentos tecnológicos, exames, procedimentos invasivos, propicia um espaço vulnerável para acontecer incidentes com a equipe e principalmente os pacientes?.

A UTI é direcionada ao tratamento e acolhimento à pacientes de alta complexidade e estado crítico, que necessitam de monitorização contínua, muitas vezes considerado um ambiente assustador para quem frequenta, assistidos com os cuidados exigidos pela sua condição clínica ${ }^{8,10}$. Na Universidade de Harvard os estudos evidenciaram que aproximadamente 42.7 milhões de incidentes com dano acontecem no mundo, por falta de segurança do paciente ${ }^{11}$.

O paciente internado na UTI está exposto a muitos riscos que ele classifica em presença de drenos, procedimentos invasivos, permanência no leito, infecção por aspiração, risco de lesão, hemorragias por desconexão de drenos e queda, tubos e cateteres, 
agitação psicomotora, desequilíbrio, padrão respiratório ineficaz, uso de ventilador mecânico e uso de sedativos ${ }^{12}$. A partir desses riscos faz necessária uma atenção dos gestores em estimular o registro e utilização dos indicadores de qualidade da assistência de enfermagem com o objetivo de implantar estratégias visando a excelência do cuidado e proporcionando ao usuário um atendimento integral ${ }^{7}$.

Diante do exposto justifica-se este trabalho de pesquisa, por entender que falhas em processos assistenciais causam incidentes nos pacientes em terapia intensiva. A partir deste estudo poderão trazer subsídios que permite contribuir para a melhoria dos processos de cuidados e uso de tecnologias nos serviços de saúde.

\section{Método}

Trata-se de um estudo retrospectivo, do tipo descritivo de abordagem quantitativa, realizado mediante coleta de dados em prontuário, nos meses de novembro e dezembro de 2016 na unidade de terapia intensiva de um Hospital Privado do município de Salvador-Bahia. O intervalo considerado para a pesquisa foi compreendido entre a admissão na UTI, permanência mínima de 24 horas, e saída desta por alta ou óbito.

A população foi formada por pessoas que estiveram internadas na unidade de terapia intensiva, no período de 01 de Junho de 2015 a 30 de Junho de 2016 , acometidos por qualquer incidente. $O$ critério de inclusão foi: Prontuários de indivíduos com internação na unidade de terapia intensiva, período igual ou superior a 24 horas. $O$ critério de exclusão foi: Prontuários não encontrados no arquivo ou faturamento. Todos os prontuários com notificações de incidentes foram avaliados, no período estudado, totalizando 210 prontuários. Destes, foram levantados 210 incidentes, sendo que em cada notificação houve um incidente. Porém, os incidentes relacionados ao erro de medicação, alguns possuíam duas notificações no mesmo prontuário. Não houve nenhuma exclusão no estudo.
A coleta de dados foi obtida a partir do levantamento do Registro de Não Conformidade (RNC) dos pacientes internados na UTI Geral do hospital de pesquisa. A partir dessa ferramenta eletrônica foi extraída uma planilha de gestão de risco com a análise de cada ocorrência, utilizada para o estudo.

Os incidentes foram classificados de acordo com a Classificação Internacional pela Segurança do Doente (CISD) em: quase evento (near miss); evento sem dano e incidente com dano (evento adverso). No evento com dano ele foi subclassificado em ligeiro, moderado, grave ou morte'.

As variáveis foram selecionadas em decorrência das falhas assistências. Definiu-se como variáveis para este estudo a ocorrência de exteriorização de dispositivos, queda, lesão por pressão, flebite e erro de medicação.

O estudo foi aprovado pelo Comitê de Ética em Pesquisa da Escola Bahiana de Medicina e Saúde Pública sob protocolo $n^{\circ} 1.779 .052$, respeitando a resolução 466/2012 sobre pesquisa envolvendo dados de seres humanos.

Os dados coletados foram digitados e processados no software Microsoft Office Excel, por meio de estatística descritiva. As variáveis foram apresentadas descritivamente em tabelas e gráficos contendo frequências absolutas ( $\mathrm{n}$ ) e relativas (\%).

O Hospital caso possui uma ferramenta para notificação de incidentes chamada Registro de Não Conformidade (RNC), iniciado no ano de 2012, quando o tema segurança do paciente passou a ser - foco maior de atenção. Ela permite que todas as categorias profissionais de saúde façam a descrição do problema, sem precisar obrigatoriamente da identificação do profissional. 


\section{Resultados}

Dos 210 incidentes notificados na unidade de terapia intensiva foram evidenciados $11 \%(n=23)$ notificações por exteriorização de dispositivos, $4,8 \%(n=10)$ por flebite. Identificou-se um total de $4,3 \%(n=9)$ referente à lesão por pressão, além de $80 \%$ ( $n=168$ ) por erro de medicação (Gráfico 1). Não houve notificações de queda.

Gráfico 1. Incidentes ocorridos e notificados na Unidade de Terapia Intensiva. Salvador, BA, 2016

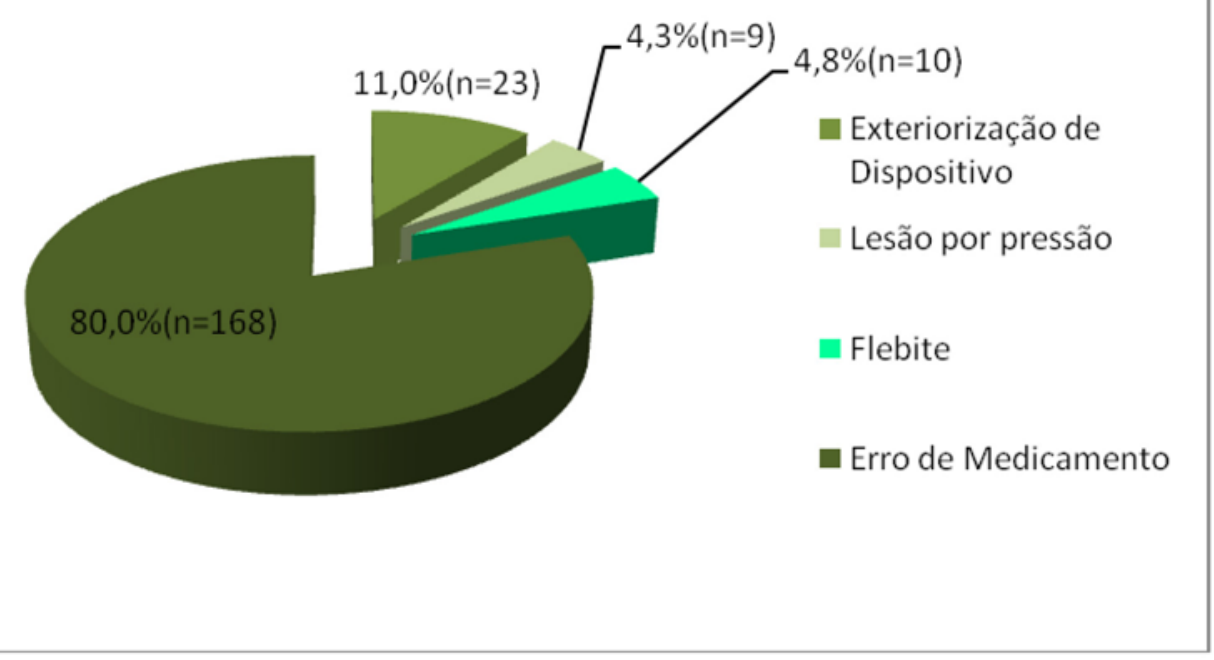

Fonte: Os autores (2019).

Com relação às 23 notificações de exteriorização de dispositivos, 18 foram incidentes sem dano e 05 notificações com dano ligeiro. A maior frequência foi de exteriorização de sonda nasoenteral (SNE), com 60,9\%. Salienta-se que um paciente exteriorizou o cateter venoso central (CVC) e a SNE no mesmo dia (Gráfico 2).

Gráfico 2. Distribuição dos incidentes notificados por exteriorização de dispositivo de acordo com o tipo de cateter. Salvador, BA, 2016

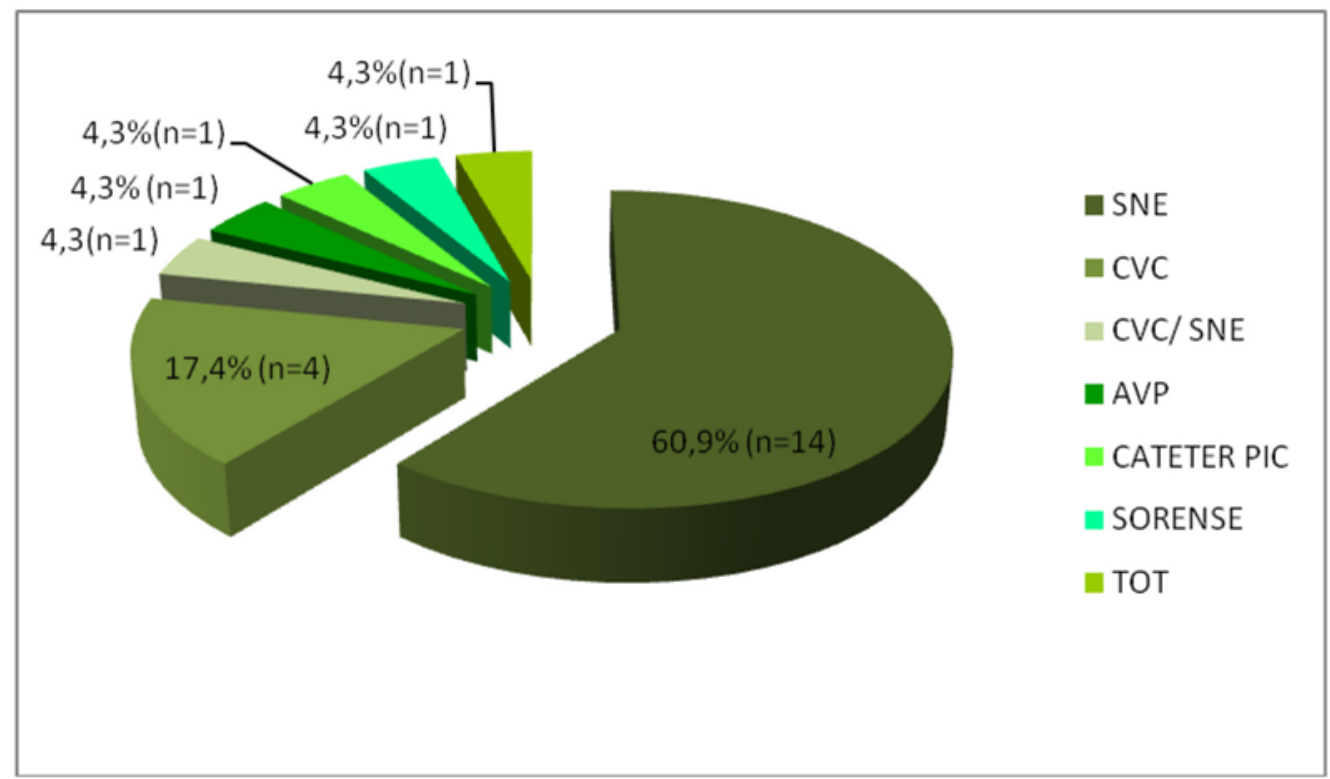

SNE: Sonda Nasoenteral; CVC: Cateter Venoso Central; AVP: ACesso Venoso Periférico; PIC: Pressão Intracraniana; TOT: Tubo Orotraqueal. Fonte: Os autores (2019). 
A faixa etária dos indivíduos que tiveram notificações por exteriorização de dispositivo está descrita na Tabela 1: 4 (17\%) foram da faixa etária $\leq 60$ anos, $11(48 \%)$ entre $61-80$ anos e $5(22 \%) \geq 81$ anos. A média de idade foi de $71 \pm 15,5$ anos.

Tabela 1. Faixa Etária dos pacientes notificados por exteriorização de dispositivo na Unidade de Terapia Intensiva. Salvador, BA, 2016

\begin{tabular}{lrr}
\hline Faixa Etária & Frequência & \multicolumn{1}{c}{$\%$} \\
\hline$\leq 60$ anos & 4 & 17,4 \\
$61-80$ & 11 & 47,8 \\
$\geq 81$ & 5 & 21,7 \\
SR $^{1}$ & 3 & 13,0 \\
\hline Total & $\mathbf{2 3}$ & 100,0 \\
\hline
\end{tabular}

Nota: ${ }^{1} \mathrm{SR}=\mathrm{Sem}$ Regis tro

Fonte: Arquivo de registro de não conformidade do lócus de estudo.

O número de notificações por flebite foi de $10(4,8 \%)$, sendo a maioria do sexo feminino $(70 \%)$. A faixa etária $\leq 60$ anos foram $3(30 \%)$ pacientes, de $61-80$ anos apenas $1(10 \%)$ e $\geq 80$ anos foram $5(50 \%)$ casos. A idade média foi de $74 \pm 19,7$ anos. Segundo a CISD, as 10 notificações foram consideradas incidente com dano ligeiro.

Quanto a lesão por pressão identificou-se um total de $9(4,3 \%)$ notificações, sendo a idade média de $77 \pm$ 15,5 anos. A divisão foi de 4 pacientes do sexo feminino, 4 do sexo masculino e um sem registro na descrição da notificação. As maiorias dos pacientes foram de idade $\geq 81$ anos (4) e com a identificação da lesão em estágio II (6) (Tabela 2). Segundo a CISD, as 9 notificações foram incidentes com dano ligeiro.

Tabela 2. Faixa Etária dos pacientes notificados com lesão por pressão na Unidade de Terapia Intensiva. Salvador, BA, 2016.

\begin{tabular}{lcc}
\hline Faixa Etária & Frequência & $\%$ \\
\hline$\leq 60$ anos & 2 & 22,2 \\
$61-80$ & 2 & 22,2 \\
$\geq 81$ & 4 & 44,4 \\
$\mathrm{SR}^{1}$ & 1 & 11,1 \\
\hline Total & $\mathbf{9}$ & $\mathbf{1 0 0 , 0}$ \\
\hline Nota : ${ }^{1}$ SR = Sem Regis tro \\
Fonte: Arquivo de registro de não conformidade do lócus de estudo.
\end{tabular}

A maioria das notificações foi por causa do Erro de Medicação (80\%), sendo 80 (48\%) pacientes do sexo masculino, 81 do sexo feminino (48\%) e 7 (4) sem registro na notificação. A maioria está dentro da faixa etária $\geq 81$ anos (72) e a idade média foi de $76 \pm 13,9$ anos. O mesmo paciente pode ter sido notificado mais de uma vez (Tabela 3). Segundo a CISD, 125 notificações foram consideradas quase evento, 42 incidentes sem danos e 1 incidente com dano ligeiro. 


\begin{tabular}{lcc}
\hline Faixa Etária & Frequência & $\%$ \\
\hline$\leq 60$ anos & 23 & 13,7 \\
$61-80$ & 66 & 39,3 \\
$\geq 81$ & 72 & 42,9 \\
$\mathrm{SR}^{1}$ & 7 & 4,2 \\
\hline Total & $\mathbf{1 6 8}$ & $\mathbf{1 0 0 , 0}$ \\
\hline Nota: ${ }^{1}$ SR $=$ Sem Registro \\
Fonte: Arquivo de registro de não conformidade do lócus de estudo.
\end{tabular}

\section{Discussão}

Das 210 notificações referidas neste estudo $80 \%$ $(n=168)$ foram por erro de medicação, sendo 125 quase eventos (não alcançou o doente), 42 incidentes sem dano (alcançou o doente, mas não resultou em prejuízo) e 1 incidente com dado ligeiro (perda de funções ou danos mínimos de curta duração). A faixa etária $\geq 81$ anos (43\%) foi mais prevalente. $O$ resultado encontrado corrobora com outros estudos.

O estudo realizado no Hospital Universitário do Sul de Minas Gerais, durante um ano, mesmo tempo desse estudo, observou 189 notificações de incidente, com $63 \%(n=119)$ por erro de medicação ${ }^{13}$. Em outra pesquisa feita na UTI adulto do Hospital Universitário em Petrolina, foram identificados 152 eventos adversos, sendo a maioria relacionado a erros de medicação, $29,6 \%(n=45)^{14}$. A qualidade da UTI está associada ao bom atendimento, traduzido com inexistência de danos, a recuperação do usuário de maneira eficiente, cultura da segurança posta em prática, execução de protocolos assistenciais, inovações e tecnologias, educação permanente e uma gestão comprometida com processos de melhorias ${ }^{9,15}$. Portanto, as falhas nos processos assistenciais podem acarretar em danos aos pacientes e o erro de medicação, é cientificamente comprovado, ser a maior causa de incidentes provocados na assistência.

No presente estudo $11 \%(n=23)$ notificações foram de exteriorização de dispositivo sendo 05 notificações com dano ligeiro, acarretando em danos mínimos ou intermediários de curta durabilidade. Podese perceber que o resultado foi elevado, assim como os outros estudos, sendo este incidente uma falha da equipe assistencial e que ocasiona, algumas vezes, a necessidade de submeter $\circ$ paciente a repetir 0 procedimento. Estudo identificou que $27,6 \%(n=42)$ dos eventos adversos na UTI estavam relacionados à extubação acidental, perda de sondas e acesso venoso central ${ }^{14}$. Em outro estudo realizado durante dois anos, no hospital privado em Salvador, em setores como UTls e internações, de 669 episódios de erros, foram identificadas 29 notificações por exteriorização de dispositivo ${ }^{16}$. $O$ que é considerado significativo quando comparado a esse estudo, que em um ano tiveram 23 notificações apenas em um setor.

A flebite teve $4,8 \%(n=10)$ notificações e todos os casos notificados, foram considerados eventos adversos com dano ligeiro. Os dados encontrados no presente estudo foram inferiores quando comparado a outras pesquisas realizadas. Este dado pode ser devido à cultura de notificações está em processo educacional. Em um estudo realizado por análise de dados secundários registrados no NOTIVISA, desde janeiro de 2007, até setembro de 2013, foram notificados 355 incidentes, sendo que $77(63,1 \%)$ ocasionou dano, sendo a flebite de maior frequência ${ }^{17}$. Em outra pesquisa, elaborada no Hospital Universitário do Sul de Minas Gerais, durante um ano, foram analisadas 189 fichas de notificações de eventos adversos, a flebite estava em segundo lugar, sendo $24,9 \%$ (47) das notificações ${ }^{13}$.

Na lesão por pressão (LP), tiveram 4,3\% $(n=9)$ notificações de evento adverso com dano ligeiro. A idade média foi de 76 anos, com a faixa etária prevalente $\geq 81$ anos, sendo a maioria das LP identificadas no estágio II. O resultado inferior, comparado aos outros estudos, pode estar relacionado ao tempo estudado e as subnotificações. A idade e o estágio das LP são semelhantes quando comparado a outras pesquisas. Em estudo realizado no hospital paulista, em 100 admissões acompanhadas, foram 
identificados 65 eventos adversos relacionados à integridade da pele, sendo $69,2 \%$ referentes a $\mathrm{LP}^{18}$. No outro estudo realizado, dos 29 (100\%) pacientes selecionados, $20(69 \%)$ apresentaram pelo menos uma $L P$, sendo que a prevalência delas foi de 42 (100\%), e destas, $23(54,8 \%)$ foi estágio II. A faixa etária de maior prevalência foi igual ou maior que 58 anos, $12(41,3 \%)^{19}$. Esse evento adverso é evitável mediante cuidados simples como mudança de decúbito do paciente de $2 / 2 h$, inspeção, proteção da pele e monitoramento ${ }^{20}$.

A queda não foi notificada, durante o período estudado da pesquisa. Porém, esse resultado não condiz com os diversos estudos publicados, podendo ser ocasionado por subnotificações da equipe, por falta de conhecimento do conceito de queda e a não fiscalização, pode ser outro fator ${ }^{13,20,21}$.

Salienta-se que diante da complexidade da unidade de terapia intensiva, o conhecimento técnico e científico é fundamental com o propósito de ofertar uma assistência à saúde de qualidade, já que cada decisão é suscetível a erros, podendo comprometer a segurança do paciente ${ }^{22}$. A qualidade da UTI está associada ao bom atendimento, traduzido com inexistência de danos, a recuperação do usuário de maneira eficiente, o comprometimento da equipe multidisciplinar, cultura da segurança posta em prática, execução de protocolos assistenciais, inovações e tecnologias, educação permanente e uma gestão comprometida com processos de melhorias ${ }^{9,15}$.

Ressalta-se como limitação do estudo o número de subnotificações e a incompletude das informações na notificação.

\section{Conclusão}

A maioria das notificações foi por causa do erro de medicação $(80 \%)$, a faixa etária no geral, mais prevalente foi $\geq 81$ anos e a divisão por sexo foi praticamente o mesmo número.

levantamento das notificações dos incidentes, mesmo existindo subnotificações, evidencia o tipo de atendimento prestado pela organização e a preocupação com a segurança do paciente. A adoção das medidas de investigação e melhoria dos processos assistências preveniu que esses incidentes evoluíssem para o evento adverso, com dano grave aos seus pacientes.

Sugere-se manter a investigação nos anos subsequentes para dar maior abrangência ao estudo e ampliar as pesquisas das notificações dos incidentes classificando por sexo e idade com análise estatística mais ampliada.

\section{Contribuição das autoras}

Lordelo MV participou da concepção, delineamento, busca e análise dos dados da pesquisa, interpretação dos resultados, redação e encaminhamento do artigo científico. Gama GGG participou da orientação da pesquisa, interpretação dos resultados, redação e conferência do artigo científico.

\section{Conflitos de interesses}

Nenhum conflito financeiro, legal ou político envolvendo terceiros (governo, empresas e fundações privadas, etc.) foi declarado para nenhum aspecto do trabalho submetido (incluindo mas não limitandose a subvenções e financiamentos, participação em conselho consultivo, desenho de estudo, preparação de manuscrito, análise estatística, etc.).

\section{Referências}

1. World Health Organization (WHO). Conceptual framework for the international classification for patient safety. [Internet]. 2009. [acesso em 2018 fev 02]. Disponível em: https://www. who.int/patientsafety/taxonomy/icps_full_report.pdf

2. Kohn LT, Corrigan JM, Donaldson MS. To err is human: building a safer health system. Washington: National Academy Press [Internet]. 2000 [acesso em $2018 \mathrm{fev}$ 02]. Disponível em: https://www.ncbi.nlm.nih.gov/ pubmed/25077248

3. Agência Nacional de Vigilância Sanitária (ANVISA). Boletim Segurança do Paciente e Qualidade em Serviços de Saúde - Incidentes Relacionados à Assistência à Saúde 2015. [Internet]. 2016 [acesso em 2018 Ago 22]. Disponível em: https://www20.anvisa.gov.br/segurancadopaciente/ index.php/publicacoes/category/boletins-estatisticos

4. Brasil. Ministério da Saúde. Portaria $n^{\circ} 529$, de $1^{\circ}$ de abril de 2013. Institui o Programa Nacional de Segurança do Paciente (PNSP). Diário Oficial da União. 2013 abr 2. 
5. Viacava F, Laguardia J, Ugà AD, Porto SM. PROADESS Avaliação de Desempenho do Sistema de Saúde Brasileiro: indicadores para monitoramento. [Internet]. 2011 . [acesso em 2018 ago 22]. Disponível em: https://www.proadess. icict.fiocruz.br/SGDPRELATORIO_FINAL\%20_com_sumario_ atualizadorev\%202014.pdf

6. Brasil. Ministério da Saúde. Agência Nacional de Vigilância Sanitária. Resolução - RDC n 36, de 25 de julho de 2013. Institui ações para a segurança do paciente em serviços de saúde e dá outras providências. 2013.

7. Lobão WM, Menezes IG. Construção, validação e normatização da escala de predisposição à ocorrência de Eventos Adversos (EPEA). Rev Latino-Am Enfermagem. 2012;20(4). doi: $10.1590 /$ S0104-11692012000400021

8. Carrias FMS, Sousa GM, Pinheiro JDS, Lustosa MA, Pereira MCC, Guimarães AEV et al. Humanized visit in an intensive care unit: a multidisciplinary look. Tempus, actas de saúde colet. $2018 ; 11$ (2):103-112. doi: 10.18569/tempus. v10i4.1966

9. Santos EV. Influência da gestão de processos administrativos na segurança de pacientes internados em unidades de terapia intensiva [dissertação]. São Paulo: Universidade Nove de Julho; 2014.

10. Oliveira $A B F$, Dias $O M$, Mello $M M$, Araújo $S$, Dragosavac $D$, Nucci $A$ et al. Fatores associados à maior mortalidade e tempo de internação prolongado em uma unidade de terapia intensiva de adultos. Rev Bras Ter Intensiva. 2010;22(3):250256. doi: $10.1590 /$ S0103-507X2010000300006

11. Jha AK, Larizgoitia I, Audera-Lopez C, PrasopaPlaizier N, Waters H, Bates DW. The global burden of unsafe medical care: analytic modelling of observational studies. BMJ Qual Saf. 2013;22(10):809-15. doi: $10.1136 /$ bmiqs-2012-001748

12. Carpenito LJ. Manual de diagnósticos de enfermagem. 13.ed. Porto Alegre: Artmed; 2011.

13. Silva LA, Terra FS, Macedo FRM, Santos SVM., Maia LG, Batista MHJ. Notification of adverse events: characterization of events occurred in a hospital institution. Rev enferm UFPE. 2014;8(9):3015-23. doi: 10.5205/1981-8963-v8i9a1002 0p3015-3023-2014

14. Souza RF, Alves AS, Alencar IGM. Eventos adversos na unidade de terapia intensiva. Rev enferm UFPE on line. 2018; 12 (1):19-27. doi: 10.5205/1981-8963-v1 2i01 a 2520 5p 19-27-2018

15. Fernandes HS, Pulzi Júnior SA, Costa Fllho R. Qualidade em terapia intensiva. Rev Bras Clín Méd. 2010;8:37-45.

16. Mascarenhas SN. Erros assistenciais e o processo de trabalho em enfermagem no hospital [dissertação]. Salvador: Universidade Federal da Bahia; 2015.
17. Lanzillotti LS, Andrade CLT, Mendes W, Seta MH. Neonatal adverse events and near misses reported in Brazil from 2007 to 2013. Cad Saúde Pública. 2016;32(9). doi: $10.1590 / 0102-311 \times 00100415$

18. Duarte SCM, Stipp MAC, Silva MM, Oliveira FT. Eventos adversos e segurança na assistência de enfermagem. Rev Bras Enferm. 2015;68(1):144-54. doi: 10.1590/00347167.2015680120p

19. Medeiros LNB, Silva DR, Guedes CDFS, Souza TKC, Araújo Neta BPA. Prevalence of pressure ulcers in intensive care units. Rev enferm UFPE on line. 2017;1 1(7):2697-703. doi: $10.5205 /$ revol.10939-97553-1-RV.1107201707

20. Lucero RJ, Lake ET, Aiken LH. Nursing care quality and adverse events in US hospitals. J Clin Nurs. 2010;19(1516):2185-95. doi: $10.1111 / \mathrm{i} .1365-2702.2010 .03250 . x$

21. Carneiro FS, Bezerra ALQ, Silva AEBC, Souza LP, Paranaguá TTB, Branquinho NCSS. Adverse events in the surgical clinic of a university hospital: a tool for assessing quality. Rev Enferm UERJ. 2011 ; 19(2):204-11.

22. Fassini $P$, Hahn GV. Riscos à segurança do paciente em unidade de internação hospitalar: concepções da equipe de enfermagem. Rev Enfermagem UFSM. 2012;2(2):290-299. doi: $10.5902 / 217976924966$ 